\title{
Tuberculous pleural effusion occurring during corticosteroid treatment of sarcoidosis
}

\author{
A J KNOX, A G WARDMAN, RL PAGE \\ From the Department of Respiratory Medicine, St James' University Hospital, Leeds
}

While the coexistence of sarcoidosis and tuberculosis is well recognised $^{1}$ and the development of pleural effusion in sarcoidosis has been described ${ }^{2-5}$ there have been no reports of the development of a tuberculous pleural effusion in a patient with sarcoidosis. We report a case in which a pleural effusion developed during corticosteroid treatment.

\section{Case report}

A 50 year old Asian woman presented initially with a short history of dyspnoea. There were no signs of note, but her chest radiograph showed bilateral hilar lymphadenopathy and diffuse interstitial shadowing. Histological examination of transbronchial biopsy specimens showed granulomatous changes suggestive of sarcoidosis, and serum angiotensin converting enzyme activity was increased at $43 \mathrm{U} / \mathrm{ml}$ (normal range 5.5-28 U/ml). Kveim and Mantoux (100 units) tests gave negative results. A diagnosis of sarcoidosis was made, and she was treated with corticosteroids. The breathlessness and radiological appearances rapidly resolved.

She continued to take prednisolone $10-15 \mathrm{mg}$ /day for the next three years, at the end of which she presented with a short history of pleuritic chest pain and was found to have a right pleural effusion (figure). Pleural biopsy showed noncaseating granulomas but the pleural aspirate contained lymphocytes in excessive numbers and acid fast bacilli, which were seen on direct examination; Mycobacterium tuberculosis was subsequently isolated by culture of the fluid. The Mantoux test (100 units) again gave a negative result. She was given a standard course of antituberculous chemotherapy while continuing corticosteroid treatment, and the effusion subsequently resolved.

\section{Discussion}

Our patient's initial illness with bilateral hilar lymphadenopathy, bilateral pulmonary infiltrates, raised serum angiotensin converting enzyme activity, and non-caseating granulomas revealed by lung biopsy, and a prompt clinical and radiological response to corticosteroid treatment, certainly suggests a diagnosis of sarcoidosis. ${ }^{6}$ Pleural effusion in sarcoidosis, while recognised, is uncommon, with a prevalence of $0-5 \% .^{2-5}$ The culture of tubercle bacilli from the

\footnotetext{
Address for reprint requests: Dr A J Knox, Department of Respiratory Medicine, St James' University Hospital, Leeds LS97TF.
}

Accepted 13 January 1986

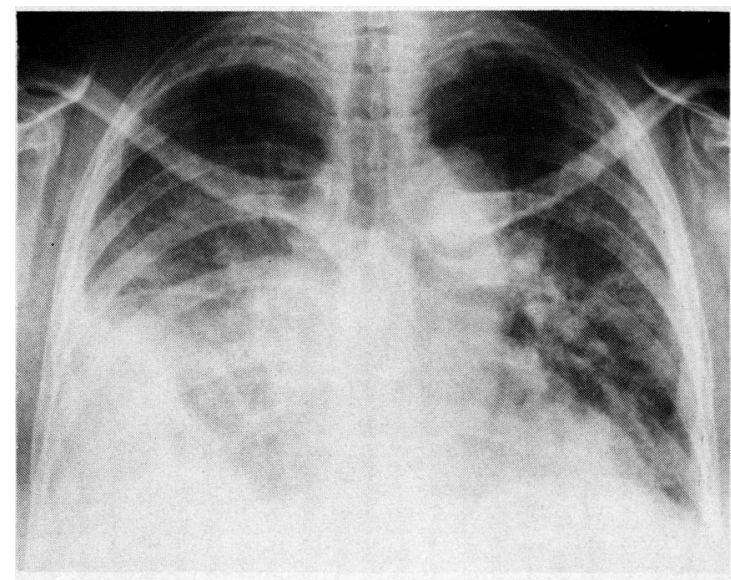

Penetrated posteroanterior chest radiograph showing the pleural effusion.

pleural fluid and the response to antituberculous treatment suggests that our patient's effusion was tuberculous rather than due to sarcoidosis. The prompt response of her initial illness to corticosteroid treatment is further evidence that the whole illness was not a manifestation of tuberculous infection. It is unusual for patients with sarcoidosis to develop a pleural effusion while having corticosteroid treatment, and the occurrence of one should prompt a diligent search for M tuberculosis.

\section{References}

1 Scadding JG, Mitchell DN. Sarcoidosis. London: Chapman and Hall, 1985.

2 Chusid EL, Sitzbach LE. Sarcoidosis of the pleura. Ann Intern Med 1974;81:190-4.

3 Wilen SB, Rabinowitz JG, Ulreich S, Lyons HA. Pleural involvement in sarcoidosis. Am J Med 1974;57:200-9.

4 Sharma OMP, Gordonson J. Pleural effusion in sarcoidosis: a report of 6 cases. Thorax 1975;30:95-101.

5 Nicholls AJ, Friend JR, Legge JS. Sarcoid pleural effusion: 3 cases and review of the literature. Thorax 1980;35:277-81.

6 James DG. Description of sarcoidosis. Report of the subcommittee on classification and definition. Ann NY Acad Sci 1976;278:742. 